\title{
Approximate Method of Riemann-Hilbert Problem for Elliptic Complex Equations of First Order in Multiply Connected Unbounded Domains
}

\author{
Guochun Wen \\ LMAM, School of Mathematical Sciences, Peking University, Beijing, China \\ Email: wengc@math.pku.edu.cn
}

Received September 26, 2012; revised November 2, 2012; accepted November 9, 2012

\begin{abstract}
In this article, we discuss the approximate method of solving the Riemann-Hilbert boundary value problem for nonlinear uniformly elliptic complex equation of first order

$$
w_{\bar{z}}=F\left(z, w, w_{z}\right) \text { in } D,
$$

with the boundary conditions

$$
\operatorname{Re}[\overline{\lambda(t)} w(t)]=r(t) \text { on } \Gamma
$$

in a multiply connected unbounded domain $D$, the above boundary value problem will be called Problem A. If the complex Equation (0.1) satisfies the conditions similar to Condition $\mathrm{C}$ of (1.1), and the boundary condition (0.2) satisfies the conditions similar to (1.5), then we can obtain approximate solutions of the boundary value problems $(0.1)$ and $(0.2)$. Moreover the error estimates of approximate solutions for the boundary value problem is also given. The boundary value problem possesses many applications in mechanics and physics etc., for instance from (5.114) and (5.115), Chapter VI, [1], we see that Problem A of (0.1) possesses the important application to the shell and elasticity.
\end{abstract}

Keywords: Approximate Method; Riemann-Hilbert Problem; Nonlinear Elliptic Complex Equations; Multiply Connected Unbounded Domains

\section{Formulation of Elliptic Equations and Boundary Value Problem}

Let $D$ be an $(N+1)$-connected domain including the infinite point with the boundary $\Gamma=\bigcup_{j=0}^{N} \Gamma_{j}$ in $\mathbb{C}$, where $\Gamma \in C_{\mu}^{2}(0<\mu<1)$. Without loss of generality, we assume that $D$ is a circular domain in $|z|>1$, where the boundary consists of $N+1$ circles

$\Gamma_{0}=\Gamma_{N+1}=\{|z|=1\}, \quad \Gamma_{j}=\left\{\left|z-z_{j}\right|=r_{j}\right\}, j=1, \cdots, N$

and $z=\infty \in D$. In this article, the notations are as the same in References [1-6]. We discuss the nonlinear uniformly elliptic complex equation of first order

$$
\left\{\begin{array}{l}
w_{\bar{z}}=F\left(z, w, w_{z}\right), \\
F=Q_{1} w_{z}+Q_{2} \bar{w}_{\bar{z}}+A_{1} w+A_{2} \bar{w}+A_{3}, \\
Q_{j}=Q_{j}\left(z, w, w_{z}\right), j=1,2, \\
A_{j}=A_{j}(z, w), j=1,2,3,
\end{array}\right.
$$

which is the complex form of the real nonlinear elliptic system of first order equations

$$
\Phi_{j}\left(x, y, u, v, u_{x}, u_{y}, v_{x}, v_{y}\right)=0, j=1,2
$$

under certain conditions (see [3]). Suppose that the complex Equation (1.1) satisfies the following conditions, namely

Condition C: 1) $Q_{j}(z, w, U)(j=1,2), A_{j}(z, w)$ $(j=1,2,3)$ are measurable in $z \in D$ for all continuous functions $w(z)$ on $\bar{D}$ and all measurable functions $U(z) \in L_{p_{0}, 2}(\bar{D})$, and satisfy

$$
L_{p, 2}\left[A_{j}, \bar{D}\right] \leq k_{0}, j=1,2, L_{p, 2}\left[A_{3}, \bar{D}\right] \leq k_{1},
$$

where $p_{0}, p\left(2<p_{0} \leq p\right), k_{0}, k_{1}$ are non-negative constants.

2) The above functions are continuous in $w \in \mathbb{C}$ for almost every point $z \in D, U \in \mathbb{C}$ and $Q_{j}=0(j=1,2), A_{j}=0(j=1,2,3)$ for $z \notin D$.

3) The complex Equation (1.1) satisfies the uniform ellipticity condition, i.e. for any $U_{1}, U_{2} \in \mathbb{C}$, the following inequality in almost every point $z \in D$ holds:

$$
\left|F\left(z, w, U_{1}\right)-F\left(z, w, U_{2}\right)\right| \leq q_{0}\left|U_{1}-U_{2}\right|,
$$


in which $q_{0}(<1)$ is a non-negative constant.

Problem A: The Riemann-Hilbert boundary value problem for the complex Equation (1.1) may be formulated as follows: Find a continuous solution $w(z)$ of (1.1) on $\bar{D}$ satisfying the boundary condition

$$
\operatorname{Re}[\overline{\lambda(z)} w(z)]=r(z), z \in \Gamma,
$$

where $|\lambda(z)|=1, z \in \Gamma$, and $\lambda(z), r(z)$ satisfy the conditions

$$
C_{\alpha}[\lambda(z), \Gamma] \leq k_{0}, C_{\alpha}[r(z), \Gamma] \leq k_{2},
$$

in which $\alpha(0<\alpha<1), k_{0}, k_{2}$ are non-negative constants.

This boundary value problem for (1.1) with $A_{3}(z, w)=0, z \in D, w \in \mathbb{C}$ and $r(z)=0, z \in \Gamma$ will be called Problem $A_{0}$. The integer

$$
K=\frac{1}{2 \pi} \Delta_{\Gamma} \arg \lambda(z)
$$

is called the index of Problem $\mathrm{A}$ and Problem $\mathrm{A}_{0}$.

Due to when the index $K<0$, Problem A may not be solvable, when $K \geq 0$, the solution of Problem A is not necessarily unique. Hence we put forward some well posednesses of Problem A with modified boundary conditions.

Problem $\mathbf{B}_{1}$ : Find a continuous solution $w(z)$ of the complex Equation (1.1) in $\bar{D}$ satisfying the boundary condition

$$
\operatorname{Re}[\overline{\lambda(z)} w(z)]=r(z)+h(z), z \in \Gamma,
$$

where

$$
h(z)=\left\{\begin{array}{ll}
0, z \in \Gamma, & \text { if } K \geq N, \\
h_{j}, z \in \Gamma_{j}, j=1, \cdots, N-K, \\
0, z \in \Gamma_{j}, j=N-K+1, \cdots, N+1,
\end{array}\right\} \text { if } 0 \leq K<N,
$$

in which $h_{j}(j=0,1, \cdots, N), h_{m}^{ \pm}(m=1, \cdots,-K-1, K<0)$ are unknown real constants to be determined appropriately. In addition, we may assume that the solution $w(z)$ satisfies the following side conditions (point conditions)

$$
\begin{aligned}
& \operatorname{Im}\left[\overline{\lambda\left(a_{j}\right)} w\left(a_{j}\right)\right]=b_{j}, \\
& j \in J= \begin{cases}1, \cdots, 2 K-N+1, & \text { if } K \geq N, \\
N-K+1, \cdots, N+1, & \text { if } 0 \leq K<N,\end{cases}
\end{aligned}
$$

where

$$
\begin{aligned}
& a_{j} \in \Gamma_{j}(j=1, \cdots, N), \\
& a_{j} \in \Gamma_{0}(j=N+1, \cdots, 2 K-N+1, K \geq N)
\end{aligned}
$$

are distinct points, and $b_{j}(j \in J)$ are all real constants satisfying the conditions

$$
\left|b_{j}\right| \leq k_{3}, j \in J
$$

herein $k_{3}$ is a nonnegative constant.

Now, we give the second well posed-ness of Problem A.

Problem $\mathbf{B}_{2}$ : If the point condition (1.9) in Problem $B_{1}$ is replaced by the integral conditions

$$
L_{j}(\bar{\lambda} w)=\left\{\begin{array}{l}
\operatorname{Im} \int_{\Gamma_{j}} \overline{\lambda(z)} w(z) \mathrm{d} s=B_{j}, \\
j=\left\{\begin{array}{l}
N-K+1, \cdots, N+1 \text { if } 0 \leq K<N, \\
1, \cdots, N+1
\end{array} \quad \text { if } K \geq N,\right. \\
\operatorname{Re} \int_{\Gamma_{0}} z^{j-N-1} \overline{\lambda(z)} w(z) \mathrm{d} s=B_{j}, \\
j=N+2, \cdots, K+1 \\
\operatorname{Im} \int_{\Gamma_{0}} z^{j-K-1} \overline{\lambda(z)} w(z) \mathrm{d} s=B_{j}, \\
j=K+2, \cdots, 2 K-N+1
\end{array}\right\} \text { if } K \geq N,
$$

respectively, where $B_{j}(j \in J)$ are real constants satisfying the conditions

$$
\left|B_{j}\right| \leq k_{3}, j \in J,
$$

in which $k_{3}$ is a nonnegative real constant.

For convenience, we sometimes will subsume the integral conditions or the point conditions under boundary conditions.

\section{A Priori Estimates of Solutions of Boundary Value Problem}

First of all, we give a representation theorem of solutions for Problem $\mathrm{B}_{1}$ and for Problem $\mathrm{B}_{2}$.

Theorem 2.1. Suppose that the complex Equation (1.1) satisfies Condition $\mathrm{C}$, and $w(z)$ is any solution of Problem $\mathrm{B}_{1}$ (or Problem $\mathrm{B}_{2}$ ) for (1.1). Then $w(z)$ is representable by

$$
w(z)=\Phi[\zeta(z)] \mathrm{e}^{\phi(z)}+\psi(z),
$$

where $\zeta(z)$ is a homeomorphism on $\bar{D}$, which quasiconformally maps $D$ onto an $(N+1)$-connected circular domain $G$ with boundary $L=\bigcup_{j=0}^{N} L_{j}$ where the $L_{j}=\zeta\left(\Gamma_{j}\right)(j=1, \cdots, N)$ are located in $|\zeta|>1$ by $L_{0}=\zeta\left(\Gamma_{0}\right)=\{|\zeta|=1\}$, and $\zeta(\infty)=\infty, \Phi(\zeta)$ is an analytic function in $G, \psi(z), \phi(z), \zeta(z)$ and its inverse function $z(\zeta)$ satisfy the estimates 


$$
\begin{gathered}
C_{\beta}[\tau, \bar{D}] \leq k_{4}, \tau=\psi(z), \varphi(z), \zeta(z) / z, \\
C_{\beta}[z(\zeta) / \zeta, \bar{G}] \leq k_{4}, \\
L_{p_{0}, 2}\left[\left|\psi_{\bar{z}}\right|+\left|\psi_{z}\right|, \bar{D}\right] \leq k_{4}, \\
L_{p_{0}, 2}\left[\left|\phi_{\bar{\zeta} \mid}\right|+\left|\phi_{\zeta}\right|, \bar{G}\right] \leq k_{4}, \\
L_{p_{0}, 2}\left[\left|\zeta_{\bar{z}}\right|+\left|\zeta_{z}\right|, \bar{D}\right] \leq k_{5}, \\
L_{p_{0}, 2}\left[\left|z_{\bar{\zeta}}\right|+\left|z_{\zeta}\right|, \bar{G}\right] \leq k_{5},
\end{gathered}
$$

in which $\beta=\min \left(\alpha, 1-2 / p_{0}\right), p_{0}\left(2<p_{0} \leq p\right), \quad k_{4}, k_{5}$ are non-negative constants,

$k_{j}=k_{j}\left(q_{0}, p_{0}, k_{0}, k_{1}, D\right), j=4,5$.

Proof. Similarly to Theorem 2.4, Chapter 2 in [3], we substitute the solution $w(z)$ of Problem $\mathrm{B}_{1}$ (or Problem $\mathrm{B}_{2}$ ) into the coefficients of the complex Equation (1.1) and consider the following system

$$
\begin{gathered}
\psi_{\bar{z}}=Q \psi_{z}+A_{1} \psi+A_{2} \bar{\psi}+A_{3}, \\
Q= \begin{cases}Q_{1}+Q_{2} \bar{w}_{\bar{z}} / w_{z} & \text { for } w_{z} \neq 0, \\
0 & \text { for } w_{z}=0 \text { or } z \notin D,\end{cases} \\
\phi_{\bar{z}}=Q \phi_{z}+A, \\
A= \begin{cases}A_{1}+A_{2} \bar{w} / w & \text { for } w(z) \neq 0, \\
0 & \text { for } w(z)=0 \text { or } z \notin D,\end{cases} \\
W_{\bar{z}}=Q W_{z}, W(z)=\Phi[\zeta(z)] .
\end{gathered}
$$

By using the continuity method and the principle of contracting mappings, we can find the solutions

$$
\begin{aligned}
& \psi(z)=\hat{T} f, \phi=\hat{T} g, \zeta(z)=\Psi[\chi(z)], \\
& \chi(z)=1 / z+\hat{T} h,
\end{aligned}
$$

where $f(z), g(z), h(z) \in L_{p_{0}, 2}(\bar{D}), 2<p_{0} \leq p, \chi(z)$ is a homeomorphism on $\bar{D}, \Psi(\chi)$ is a univalent analytic function, which conformally maps $E=\chi(D)$ onto an $(N+1)$-connected circular domain $G$, and $\Phi(\zeta)$ is an analytic function in $G$. We can verify that $\psi(z), \phi(z)$ satisfy the estimates (2.2) and (2.3). Moreover noting that $\zeta(z)$ is a homeomorphic solution of the Beltrami complex Equation (2.7), which maps the circular domain $D$ onto the circular domain $G$ with the condition $\infty=\zeta(\infty)$ and $L_{0}=\zeta\left(\Gamma_{0}\right)=\{|\zeta|=1\}$, in accordance with the result in Lemma 2.1, Chapter 2, [3], we see that the estimate (2.4) is true.

Now, we derive a priori estimates of solutions for Problem $B_{1}$ and for Problem $B_{2}$ for the complex Equation (1.1).

Theorem 2.2. Under the same conditions as in Theorem 2.1, any solution $w(z)$ of Problem $\mathrm{B}_{1}$ (or Problem $B_{2}$ ) for (1.1) satisfies the estimates

$$
\begin{gathered}
C_{\eta}[w(z), \bar{D}] \leq M_{1}=M_{1}\left(q_{0}, p_{0}, k, \alpha, K, D\right), \\
L_{p_{0}, 2}\left[\left|w_{\bar{z}}\right|+\left|w_{z}\right|, \tilde{D}\right] \leq M_{2} \\
=M_{2}\left(q_{0}, p_{0}, k, \alpha, K, \tilde{D}\right),
\end{gathered}
$$

where

$$
k=k\left(k_{0}, k_{1}, k_{2}, k_{3}\right), \eta=\alpha \beta^{2}, 2<p_{0} \leq p, M_{j}(j=1,2)
$$

are non-negative constants only dependent on $q_{0}, p_{0}, k, \alpha, K, D$ and $q_{0}, p_{0}, k, \alpha, K, \tilde{D}$ respectively.

Proof. On the basis of Theorem 2.1, the solution $w(z)$ of Problem $\mathrm{B}_{1}$ (or Problem $\mathrm{B}_{2}$ ) can be expressed the formula as in (2.1), hence the boundary value problem $\mathrm{B}_{1}$ can be transformed into the boundary value problem (Problem $\tilde{\mathrm{B}}$ ) for analytic functions

$$
\begin{array}{r}
\operatorname{Re}[\overline{\Lambda(\zeta)} \Phi(\zeta)]=R(\zeta)+H(\zeta), \zeta \in L, \quad \text { (2.11) } \\
H(\zeta)=\left\{\begin{array}{l}
0, \zeta \in L, \\
h_{j}, \zeta \in L_{j}, j=1, \cdots, N-K, \\
0, \zeta \in L_{j}, j=N-K+1, \cdots, N+1, \\
h_{j}, \zeta \in L_{j}, j=1, \cdots, N, \\
h_{0}+\operatorname{Re} \sum_{m=1}^{-K-1}\left(h_{m}^{+}+\mathrm{i} h_{m}^{-}\right) \zeta^{m}, z \in L_{0},
\end{array}\right\} \text { if } 0 \leq K<N \\
\operatorname{Im}\left[\overline{\Lambda\left(a_{j}^{\prime}\right)} \Phi\left(a_{j}^{\prime}\right)\right]=b_{j}^{\prime}, j \in J,
\end{array}
$$

where

$$
\begin{aligned}
& \overline{\Lambda(\zeta)}=\overline{\lambda[z(\zeta)]} \mathrm{e}^{\phi[z(\zeta)]}, \\
& R(\zeta)=r[z(\zeta)]-\operatorname{Re}\{\overline{\lambda[z(\zeta)] \psi} \psi[z(\zeta)]\}, \\
& a_{j}^{\prime}=\zeta\left(a_{j}\right), b_{j}^{\prime}=b_{j}-\operatorname{Im}\left[\overline{\lambda\left(a_{j}\right)} \psi\left(a_{j}\right)\right], j \in J .
\end{aligned}
$$

By (2.2)-(2.4), it can be seen that $\Lambda(\zeta), R(\zeta), b_{j}^{\prime}(j \in J)$ satisfy the conditions

$$
\begin{aligned}
& C_{\alpha \beta}[\Lambda(\zeta), L] \leq M_{3}, C_{\alpha \beta}[R(\zeta), L] \leq M_{3}, \\
& \left|b_{j}^{\prime}\right| \leq M_{3}, j \in J,
\end{aligned}
$$

where $M_{3}=M_{3}\left(q_{0}, p_{0}, k, \alpha, K, D\right)$. If we can prove that the solution $\Phi(\zeta)$ of Problem B satisfies the estimate

$$
C_{\alpha \beta}[\Phi(\zeta), \bar{G}] \leq M_{4}, C_{\alpha \beta}\left[\Phi^{\prime}(\zeta), \tilde{G}\right] \leq M_{5},
$$

in which $\tilde{D}=\{z \in D \mid \operatorname{dist}(z, \Gamma) \geq \varepsilon>0\}, \tilde{G}=\zeta(\tilde{D})$, $M_{4}=M_{4}\left(q_{0}, p_{0}, k, \alpha, K, D\right)$,

$M_{5}=M_{5}\left(q_{0}, p_{0}, k, \alpha, K, \tilde{D}\right)$, then from the representation (2.1) of the solution $w(z)$ and the estimates (2.2)-(2.4) and (2.15), the estimates (2.9) and (2.10) can be derived. 
It remains to prove that $(2.15)$ holds. For this, we first verify the boundedness of $\Phi(\zeta)$, i.e.

$$
\begin{aligned}
& C[\Phi(\zeta), \bar{G}] \leq M_{6} \\
& =M_{6}\left(q_{0}, p_{0}, k, \alpha, K, D\right) .
\end{aligned}
$$

Suppose that (2.16) is not true. Then there exist sequences of functions $\left\{\Lambda_{n}(\zeta)\right\},\left\{R_{n}(\zeta)\right\},\left\{b_{j n}^{\prime}\right\}$ satisfying the same conditions as $\Lambda(\zeta), R(\zeta), b_{j}^{\prime}$, which uniformly converge to $\Lambda_{0}(\zeta), R_{0}(\zeta), b_{j 0}^{\prime}(j \in J)$ on $L$ respectively. For the solution $\Phi_{n}(\zeta)$ of the boundary value problem (Problem $\mathrm{B}_{n}$ ) corresponding to

$\Lambda_{n}(\zeta), R_{n}(\zeta), b_{j n}^{\prime}(j \in J)$, we have

$I_{n}=C\left[\Phi_{n}(\zeta), \bar{G}\right] \rightarrow \infty$ as $n \rightarrow \infty$. There is no harm in assuming that $I_{n} \geq 1, n=1,2, \cdots$. Obviously $\tilde{\Phi}_{n}(\zeta)=\Phi_{n}(\zeta) / I_{n} \quad$ satisfies the boundary conditions

$$
\begin{gathered}
\operatorname{Re}\left[\overline{\Lambda_{n}(\zeta)} \Phi_{n}(\zeta)\right] \\
=\left[R_{n}(\zeta)+H(\zeta)\right] / I_{n}, \zeta \in L, \\
\operatorname{Im}\left[\overline{\Lambda_{n}\left(a_{n}^{\prime}\right)} \tilde{\Phi}_{n}\left(a_{n}^{\prime}\right)\right]=b_{j n}^{\prime} / I_{n}, j \in J .
\end{gathered}
$$

Applying the Schwarz formula, the Cauchy formula and the method of symmetric extension (see Theorem 1.4, Chapter 1, [3]), the estimates

$$
C_{\alpha \beta}\left[\tilde{\Phi}_{n}(\zeta), \bar{G}\right] \leq M_{7}, C\left[\tilde{\Phi}_{n}^{\prime}(\zeta), \tilde{G}\right] \leq M_{8}
$$

can be obtained, where $M_{7}=M_{7}\left(q_{0}, p_{0}, k, \alpha, K, D\right)$, $M_{8}=M_{8}\left(q_{0}, p_{0}, k, \alpha, K, \tilde{D}\right)$. Thus we can select a subsequence of $\left\{\tilde{\Phi}_{n}(\zeta)\right\}$, which uniformly converge to an analytic function $\tilde{\Phi}_{0}(\zeta)$ in $G$, and $\tilde{\Phi}_{0}(\zeta)$ satisfies the homogeneous boundary conditions

$$
\begin{aligned}
& \operatorname{Re}\left[\overline{\Lambda_{0}(\zeta)} \Phi_{0}(\zeta)\right]=H(\zeta), \zeta \in L, \\
& \operatorname{Im}\left[\overline{\Lambda_{0}\left(a_{j}^{\prime}\right)} \tilde{\Phi}_{0}\left(a_{j}^{\prime}\right)\right]=0, j \in J .
\end{aligned}
$$

On the basis of the uniqueness theorem (see Theorem $2.4)$, we conclude that $\tilde{\Phi}_{0}(\zeta)=0, \zeta \in \bar{G}$. However, from $C\left[\tilde{\Phi}_{n}(\zeta), \bar{G}\right]=1$, it follows that there exists a point $\zeta_{*} \in \bar{G}$, such that $\left|\tilde{\Phi}_{0}\left(\zeta_{*}\right)\right|=1$. This contradiction proves that (2.16) holds. Afterwards using the method which leads from $C\left[\tilde{\Phi}_{n}(\zeta), \bar{G}\right]=1$ to $(2.19)$, the estimate (2.15) can be derived.

Similarly, we can verify that any solution $w(z)$ of Problem $\mathrm{B}_{2}$ satisfies the estimates (2.9) and (2.10).

Theorem 2.3. Under the same conditions as in Theorem 2.1, any solution $w(z)$ of Problem $\mathrm{B}_{1}$ (or Problem $B_{2}$ ) for (1.1) satisfies

$$
\begin{aligned}
& C_{\eta}[w(z), \bar{D}] \leq M_{9} k_{*}, \\
& L_{p_{0}, 2}\left[\left|w_{\bar{z}}\right|+\left|w_{z}\right|, \tilde{D}\right] \leq M_{10} k_{*},
\end{aligned}
$$

where $\eta, p_{0}, \tilde{D}$ are as stated in Theorem 2.2,

$$
k_{*}=k_{1}+k_{2}+k_{3}, M_{j}=M_{j}\left(q_{0}, p_{0}, k_{0}, \alpha, K, D\right), j=9,10 \text {. }
$$

Proof. If $k_{*}=0$, i.e. $k_{1}=k_{2}=k_{3}=0$, from Theorem 2.4 , it follows that $w(z)=0, z \in D$. If $k_{*}>0$, it is easy to see that $W(z)=w(z) / k_{*}$ satisfies the complex equation and boundary conditions

$$
\begin{array}{r}
W_{\bar{z}}-Q_{1} W_{z}-Q_{2} \bar{W}_{\bar{z}}-A_{1} W-A_{2} \bar{W}=A_{3} / k_{*}, z \in D, \\
\operatorname{Re}[\overline{\lambda(z)} W(z)]=[r(z)+h(z)] / k_{*}, z \in \Gamma, \\
\operatorname{Im}\left[\overline{\lambda\left(a_{j}\right)} W\left(a_{j}\right)\right]=b_{j} / k_{*}, \\
\left(\text { or } L_{j} \bar{\lambda} W=B_{j} / k_{*}\right), j \in J,
\end{array}
$$

Noting that

$$
\begin{aligned}
& L_{p, 2}\left[A_{3} / k_{*}, \bar{D}\right] \leq 1, C_{\alpha}\left[r(z) / k_{*}, \Gamma\right] \leq 1, \\
& \left|b_{j} / k_{*}\right| \leq 1\left(\text { or }\left|B_{j} / k_{*}\right| \leq 1\right), j \in J
\end{aligned}
$$

and according to the proof of Theorem 2.2, we have

$$
\begin{aligned}
& C_{\eta}[W(z), \bar{D}] \leq M_{9}, \\
& L_{p_{0}, 2}\left[\left|W_{\bar{z}}\right|+\left|W_{z}\right|, \tilde{D}\right] \leq M_{10} .
\end{aligned}
$$

From the above estimates, it immediately follows that (2.22) holds.

Next, we prove the uniqueness of solutions of Problem $\mathrm{B}_{1}$ and Problem $\mathrm{B}_{2}$ for the complex Equation (1.1). For this, we need to add the following condition: For any continuous functions $w_{1}(z), w_{2}(z)$ on $\bar{D}$ and $U(z) \in L_{p_{0}, 2}(\bar{D})$, there is

$$
\begin{aligned}
& F\left(z, w_{1}, U\right)-F\left(z, w_{2}, U\right) \\
& =A\left(z, w_{1}, w_{2}, U\right)\left(w_{1}-w_{2}\right),
\end{aligned}
$$

where $A\left(z, w_{1}, w_{2}, U\right) \in L_{p_{0}, 2}(\bar{D})$. When (1.1) is linear, (3.27) obviously holds.

Theorem 2.4. If Condition $C$ and (2.27) hold, then the solution of Problem $\mathrm{B}_{1}$ (or Problem $\mathrm{B}_{2}$ ) for (1.1) is unique.

Proof. Let $w_{1}(z), w_{2}(z)$ be two solutions of Problem $\mathrm{B}_{1}$ for (1.1). By Condition $\mathrm{C}$ and (2.27), we see that $w(z)=w_{1}(z)-w_{2}(z)$ is a solution of the following boundary value problem

$$
\begin{gathered}
w_{\bar{z}}-\tilde{Q} w_{z}=\tilde{A} w, z \in D, \\
\operatorname{Re}[\overline{\lambda(z)} w(z)]=h(z), z \in \Gamma, \\
\operatorname{Im}\left[\overline{\lambda\left(a_{j}\right)} w\left(a_{j}\right)\right]=0, j \in J,
\end{gathered}
$$


where

$$
\begin{aligned}
& \tilde{Q}=\left\{\begin{array}{l}
{\left[F\left(z, w_{1}, w_{1 z}\right)-F\left(z, w_{1}, w_{2 z}\right)\right] /\left(w_{1}-w_{2}\right)_{z}} \\
\text { for } w_{1 z} \neq w_{2 z}, \\
0 \text { for } w_{1 z}=w_{2 z}, z \in D,
\end{array}\right. \\
& \tilde{A}=\left\{\begin{array}{l}
{\left[F\left(z, w_{1}, w_{2 z}\right)-F\left(z, w_{2}, w_{2 z}\right)\right] /\left(w_{1}-w_{2}\right)} \\
\text { for } w_{1}(z) \neq w_{2}(z), \\
0 \text { for } w_{1}(z)=w_{2}(z), z \in D,
\end{array}\right.
\end{aligned}
$$

and $|\tilde{Q}| \leq q_{0}<1, z \in D, L_{p_{0}, 2}(\tilde{A}, \bar{D})<\infty$. According to the representation (2.1), we have

$$
w(z)=\Phi[\zeta(z)] \mathrm{e}^{\phi(z)},
$$

where $\phi(z), \zeta(z), \Phi(\zeta)$ are as stated in Theorem 2.1 . It can be seen that the analytic function $\Phi(z)$ satisfies the boundary conditions of Problem $\mathrm{B}_{0}$ :

$$
\begin{gathered}
\operatorname{Re}[\overline{\Lambda(\zeta)} \Phi(\zeta)]=H(\zeta), \zeta \in L=\zeta(\Gamma), \\
\operatorname{Im}\left[\overline{\Lambda\left(a_{j}^{\prime}\right)} \Phi\left(a_{j}^{\prime}\right)\right]=0, j \in J
\end{gathered}
$$

where $\Lambda(\zeta), H(\zeta)(\zeta \in L), a_{j}^{\prime}(j \in J)$ are as stated in (2.11)-(2.13). In accordance with Theorem 2.2, it can be derived that $\Phi(\zeta)=0, \zeta \in G=\zeta(D)$. Hence,

$$
w(z)=\Phi[\zeta(z)] \mathrm{e}^{\phi(z)}=0 \text {, i.e. } w_{1}(z)=w_{2}(z), z \in D \text {. }
$$

\section{The Continuity Method of Solving Boundary Value Problem}

Next, we discuss the modified Riemann-Hilbert boundary value problems (Problem $\mathrm{B}_{1}$ and Problem $\mathrm{B}_{2}$ ) for the nonlinear elliptic complex Equation (1.1) in the $(N+1)$-connected unbounded domain $D$ as stated in Section 1, here we use the Newton imbedding method of another form and give an error estimate, which is better than that as stated before. In the following, we only deal with Problem $B_{1}$, because by using the same method, Problem $\mathrm{B}_{2}$ can be discussed.

Theorem 3.1. Suppose that the nonlinear elliptic Equation (1.1) satisfies Condition $C$ and (1.6), (1.10), on $\bar{D}$. Then Problem $\mathrm{B}_{1}$ for (1.1) has a solution $w(z) \in W_{p_{0}, 2}^{1}(D)$.

Proof We introduce the nonlinear elliptic complex equation with the parameter $t \in[0,1]$ :

$$
w_{\bar{z}}-t F\left(z, w, w_{z}\right)=A(z),
$$

where $A(z)$ is any measurable function in $D$ and $A(z) \in L_{p_{0}, 2}(\bar{D}), 2<p_{0} \leq p$. When $t=0$, it is not difficult to see that there exists a unique solution $w(z)$ of Problem $\mathrm{B}_{1}$ for the complex Equation (3.1), which possesses the form

$$
\begin{aligned}
& w(z)=\Phi(z)+\psi(z), \\
& \psi(z)=\hat{T} A=-\frac{1}{\pi} \iint_{D} \frac{A(1 / \zeta)}{\bar{\zeta}^{2} \zeta(1-\zeta z)} \mathrm{d} \sigma_{\zeta},
\end{aligned}
$$

where $\Phi(z)$ is an analytic function in $D$ and satisfies the boundary conditions

$$
\begin{aligned}
& \operatorname{Re}[\overline{\lambda(z)} \Phi(z)]=r(z)-\operatorname{Re}[\overline{\lambda(z)} \psi(z)]+h(z), \\
& z \in \Gamma \\
& \quad \operatorname{Im}\left[\overline{\lambda\left(a_{j}\right)} \Phi\left(a_{j}\right)\right]=b_{j}-\operatorname{Im}\left[\overline{\lambda\left(a_{j}\right)} \psi\left(a_{j}\right)\right], \\
& \quad j \in J .
\end{aligned}
$$

From Theorem Theorem 2.2, We see that

$$
\begin{aligned}
& w(z) \in B=C_{\beta}(\bar{D}) \cap W_{p_{0}, 2}^{1}(D), \\
& \beta=\alpha\left[\min \left(\alpha, 1-2 / p_{0}\right)\right]^{2} .
\end{aligned}
$$

Suppose that when $t=t_{0}\left(0 \leq t_{0}<1\right)$, Problem $\mathrm{B}_{1}$ for the complex Equation (1.18) has a unique solution, we shall prove that there exists a neighborhood of $t_{0}: E=\left\{\left|t-t_{0}\right| \leq \delta, 0 \leq t \leq 1, \delta>0\right\}$, so that for every $t \in E$ and any function $A(z) \in L_{p_{0}, 2}(\bar{D})$, Problem $B_{1}$ for (1.18) is solvable. In fact, the complex Equation (3.2) can be written in the form

$$
w_{\bar{z}}-t_{0} F\left(z, w, w_{z}\right)=\left(t-t_{0}\right) F\left(z, w, w_{z}\right)+A(z) .
$$

We arbitrarily select a function $w_{0}(z) \in B=C_{\beta}(\bar{D}) \cap W_{p_{0}, 2}^{1}(D)$, in particular $w_{0}(z)=0$ on $\bar{D}$. Let $w_{0}(z)$ be replaced into the position of $w(z)$ in the right hand side of (1.22). By Condition $\mathrm{C}$, it is obvious that

$$
B_{0}(z)=\left(t-t_{0}\right) F\left(z, w_{0}, w_{0 z}\right)+A(z) \in L_{p_{0}}(\bar{D}) .
$$

Noting the (3.5) has a solution $w_{1}(z) \in B$. Applying the successive iteration, we can find out a sequence of functions: $w_{n}(z) \in B, n=1,2, \cdots$, which satisfy the complex equations

$$
\begin{aligned}
& w_{n+1 \bar{z}}-t_{0} F\left(z, w_{n+1}, w_{n+1 z}\right) \\
& =\left(t-t_{0}\right) F\left(z, w_{n}, w_{n z}\right)+A(z), n=1,2, \cdots .
\end{aligned}
$$

The difference of the above equations for $n+1$ and $n$ is as follows:

$$
\begin{aligned}
& \left(w_{n+1}-w_{n}\right)_{\bar{z}} \\
& -t_{0}\left[F\left(z, w_{n+1}, w_{n+1 z}\right)-F\left(z, w_{n}, w_{n z}\right)\right] \\
& =\left(t-t_{0}\right)\left[F\left(z, w_{n}, w_{n z}\right)-F\left(z, w_{n-1}, w_{n-1 z}\right)\right], \\
& n=1,2, \cdots .
\end{aligned}
$$

From Condition $\mathrm{C}$, on $\bar{D}$, it can be seen that 


$$
\begin{aligned}
& F\left(z, w_{n+1}, w_{n+1 z}\right)-F\left(z, w_{n}, w_{n z}\right) \\
&= F\left(z, w_{n+1}, w_{n+1 z}\right)-F\left(z, w_{n+1}, w_{n z}\right) \\
&+ {\left[F\left(z, w_{n+1}, w_{n z}\right)-F\left(z, w_{n}, w_{n z}\right)\right] } \\
&= \tilde{Q}_{n+1}(z)\left(w_{n+1}-w_{n}\right)_{z}+\tilde{A}_{n+1}(z)\left(w_{n+1}-w_{n}\right), \\
&\left|\tilde{Q}_{n+1}(z)\right| \leq q_{0}<1, \tilde{A}_{n+1}(z) \in L_{p_{0}, 2}(\bar{D}), n=1,2, \cdots,
\end{aligned}
$$

and

$$
\begin{aligned}
& L_{p_{0}, 2}\left[F\left(z, w_{n}, w_{n z}\right)-F\left(z, w_{n-1}, w_{n-1 z}\right), \bar{D}\right] \\
& \leq q_{0} L_{p_{0}, 2}\left[\left(w_{n}-w_{n-1}\right)_{z}, \bar{D}\right]+k_{0} C\left[w_{n}-w_{n-1}, \bar{D}\right] \\
& \leq\left(q_{0}+k_{0}\right) C_{\beta}\left[w_{n}-w_{n-1}, \bar{D}\right] \\
& +L_{p_{0}, 2}\left[\left|\left(w_{n}-w_{n-1}\right)_{\bar{z}}\right|+\left|\left(w_{n}-w_{n-1}\right)_{z}\right|, \bar{D}\right] \\
& =\left(q_{0}+k_{0}\right) L_{n} .
\end{aligned}
$$

Moreover, $w_{n+1}(z)-w_{n}(z)$ satisfies the homogeneous boundary conditions

$$
\begin{gathered}
\operatorname{Re}\left[\overline{\lambda(z)}\left(w_{n+1}(z)-w_{n}(z)\right)\right]=h(z), z \in \Gamma, \\
\operatorname{Im}\left[\overline{\lambda\left(a_{j}\right)}\left(w_{n+1}\left(a_{j}\right)-w_{n}\left(a_{j}\right)\right)\right]=0, j \in J .
\end{gathered}
$$

On the basis of Theorem 2.3, we have

$$
\begin{aligned}
L_{n+1} & =C_{\beta}\left[w_{n+1}-w_{n}, \bar{D}\right] \\
& +L_{p_{0}, 2}\left[\left|\left(w_{n+1}-w_{n}\right)_{z}\right|+\left|\left(w_{n+1}-w_{n}\right)_{z}\right|, \bar{D}\right] \\
& \leq M\left|t-t_{0}\right|\left(q_{0}+k_{0}\right) L_{n},
\end{aligned}
$$

where $M=M_{9}+M_{10}, M_{9}, M_{10}$ is as stated in (2.22). Provided $\delta(>0)$ is small enough, so that $\eta=\delta M\left(q_{0}+k_{0}\right)<1$, it can be obtained that

$$
\begin{aligned}
& L_{n+1} \leq \eta L_{n} \leq \eta^{n} L_{1} \\
& =\eta^{n}\left[C_{\beta}\left(w_{1}, \bar{D}\right)+L_{p_{0}, 2}\left(\left|w_{1 \bar{z}}\right|+\left|w_{1 z}\right|, \bar{D}\right)\right]
\end{aligned}
$$

for every $t \in E$. Thus

$$
\begin{aligned}
& S\left(w_{n}-w_{m}\right) \\
= & C_{\beta}\left[w_{n}-w_{m}, \bar{D}\right] \\
& +L_{p_{0}, 2}\left[\left|\left(w_{n}-w_{m}\right)_{\bar{z}}\right|+\left|\left(w_{n}-w_{m}\right)_{z}\right|, \bar{D}\right] \\
\leq & L_{n}+L_{n-1}+\cdots+L_{m+1} \\
\leq & \left(\eta^{n-1}+\eta^{n-2}+\cdots+\eta^{m}\right) L_{1} \\
= & \eta^{m}\left(1+\eta+\cdots+\eta^{n-m-1}\right) L_{1} \\
\leq & \eta^{N+1} \frac{1-\eta^{n-m}}{1-\eta} L_{1} \leq \frac{\eta^{N+1}}{1-\eta} L_{1}
\end{aligned}
$$

for $n \geq m>N$, where $N$ is a positive integer. This shows that $S\left(w_{n}-w_{m}\right) \rightarrow 0$ as $n, m \rightarrow \infty$. Following the completeness of the Banach space
$B=C_{\beta}(\bar{D}) \cap W_{p_{0}, 2}^{1}(D)$, there is a function $w_{*}(z) \in B$, such that when $n \rightarrow \infty$,

$$
\begin{aligned}
& S\left(w-w_{*}\right)=C_{\beta}\left[w_{n}-w_{*}, \bar{D}\right] \\
& +L_{p_{0}, 2}\left[\left|\left(w_{n}-w_{*}\right)_{\bar{z}}\right|++\left|\left(w_{n}-w_{*}\right)_{z}\right|, \bar{D}\right] \rightarrow 0 .
\end{aligned}
$$

By Condition $\mathrm{C}$ and (1.6), (1.10), from the above formula it follows that $W_{*}(z)$ is a solution of Problem $\mathrm{B}_{1}$ for (3.5), i.e. (3.1) for $t \in E$. It is easy to see that the positive constant $\delta$ is independent of $t_{0}\left(0 \leq t_{0}<1\right)$. Hence from Problem $B_{1}$ for the complex Equation (3.1) with $t=t_{0}=0$ is solvable, we can derive that when $t=\delta, 2 \delta, \cdots,[1 / \delta] \delta, 1$, Problem $\mathrm{B}_{1}$ for (3.1) are solvable, especially Problem $\mathrm{B}_{1}$ for (3.2) with $t=1$ and $A(z)=0$, namely Problem $B_{1}$ for (1.1) has a unique solution.

\section{Error Estimates of Approximate Solutions for Boundary Value Problem}

In this section, we shall introduce an error estimate of the above approximate solutions of the boundary value problem and can give the following error estimate of the approximate solutions.

Theorem 4.1 Let $w=w(z)$ be a solution of Problem $\mathrm{B}_{1}$ for the complex Equation (1.1) satisfying Condition $\mathrm{C}$ and (1.6), (1.10) on $\bar{D}$, and $w_{n}^{t}=w_{n}(z, t)$ be its appro- ximation as stated in the proof of Theorem 2.2 with $A(z)=(1-t) F(z, 0,0)$. Then we have the following error estimate

$$
\begin{aligned}
& S\left(w-w_{n}^{t}\right)=C_{\beta}\left(w-w_{n}^{t}, \bar{D}\right) \\
& +L_{p_{0}, 2}\left[\left|\left(w-w_{n}^{t}\right)_{\bar{z}}\right|+\left|\left(w-w_{n}^{t}\right)_{z}\right|, \bar{D}\right] \\
& \leq \gamma k\left[\frac{1-\gamma\left|t-t_{0}\right|^{n}}{1-\gamma\left|t-t_{0}\right|}(1-t)+\left(\gamma\left|t-t_{0}\right|\right)^{n}\left(1-t_{0}\right)\right],
\end{aligned}
$$

where $\gamma=M\left(q_{0}+k_{0}\right), \quad k=M\left(k_{1}+k_{2}+k_{3}\right)$, $M=M_{9}+M_{10}, M_{9}, M_{10}, q_{0} \quad$ and $k_{j}(j=0,1,2,3)$ are constants in (1.3), (1.6) and (1.10).

Proof From (1.1) and (2.23) with $A(z)=(1-t) F(z, 0,0)$, we have

$$
\begin{aligned}
& \left(w-w_{n+1}^{t}\right)_{\bar{z}}=F\left(z, w, w_{z}\right)-t_{0} F\left(z, w_{n+1}^{t}, w_{n+1 z}^{t}\right) \\
& -\left(t-t_{0}\right) F\left(z, w_{n}^{t}, w_{n z}^{t}\right)-(1-t) F(z, 0,0) \\
& =(1-t)\left[F\left(z, w, w_{z}\right)-F(z, 0,0)\right] \\
& +t_{0}\left[F\left(z, w, w_{z}\right)-F\left(z, w_{n+1}^{t}, w_{n+1 z}^{t}\right)\right] \\
& +\left(t-t_{0}\right) \times\left[F\left(z, w, w_{z}\right)-F\left(z, w_{n}^{t}, w_{n z}^{t}\right)\right] \\
& =t_{0}\left[\tilde{Q}\left(z, w, w_{z}, w_{n+1}^{t}\right)\left(w-w_{n+1}^{t}\right)_{z}\right. \\
& \left.\quad+\tilde{A}\left(z, w, w_{n+1}^{t}, w_{n+1 z}^{t}\right)\left(w-w_{n+1}^{t}\right)\right] \\
& +(1-t)\left[F\left(z, w, w_{z}\right)-F(z, 0,0)\right] \\
& +\left(t-t_{0}\right)\left[F\left(z, w, w_{z}\right)-F\left(z, w_{n}^{t}, w_{n z}^{t}\right)\right] .
\end{aligned}
$$


It is clear that $w-w_{n+1}^{t}$ satisfies the homogeneous boundary conditions

$$
\begin{aligned}
& \operatorname{Re}\left[\overline{\lambda(z)}\left(w(z)-w_{n+1}^{t}(z)\right)\right]=h(z), z \in \Gamma, \\
& \operatorname{Im}\left[\overline{\lambda\left(a_{j}\right)}\left(w\left(a_{j}\right)-w_{n+1}^{t}\left(a_{j}\right)\right)\right]=0, j \in J .
\end{aligned}
$$

\section{Noting that}

$$
\begin{gathered}
\tilde{Q}=\tilde{Q}\left(z, w, w_{z}, w_{n+1}^{t}\right), \tilde{A}=\tilde{A}\left(z, w, w_{n+1}^{t}, w_{n+1 z}^{t}\right) \text { satisfy } \\
|\tilde{Q}| \leq q_{0}<1, L_{p_{0}, 2}[\tilde{A}, \bar{D}] \leq k_{0}, \text { and } \\
\quad L_{p_{0}, 2}\left[F\left(z, w, w_{z}\right)-F(z, 0,0), \bar{D}\right] \\
\quad \leq q_{0} L_{p_{0}, 2}\left[w_{z}, \bar{D}\right]+k_{0} C[w, \bar{D}] \\
\quad \leq\left(q_{0}+k_{0}\right)\left[L_{p_{0}, 2}\left(w_{z}, \bar{D}\right)+C(w, \bar{D})\right] \\
\quad \leq\left(q_{0}+k_{0}\right) S(w), \\
L_{p_{0}, 2}\left[F\left(z, w, w_{z}\right)-F\left(z, w_{n}^{t}, w_{n z}^{t}\right), \bar{D}\right] \\
\leq q_{0} L_{p_{0}, 2}\left[\left(w-w_{n}^{t}\right)_{z}, \bar{D}\right]+k_{0} C\left[w-w_{n}^{t}, \bar{D}\right] \\
\leq\left(q_{0}+k_{0}\right)\left[L_{p_{0}, 2}\left(\left(w-w_{n}^{t}\right)_{z}, \bar{D}\right)+C\left(w-w_{n}^{t}, \bar{D}\right)\right] \\
\leq\left(q_{0}+k_{0}\right) S\left(w-w_{n}^{t}\right),
\end{gathered}
$$

and according to Theorem 2.2, it can be concluded

$$
\begin{aligned}
& S\left(w-w_{n+1}^{t}\right) \\
\leq & M\left[(1-t)\left(q_{0}+k_{0}\right) S(w)+\left|t-t_{0}\right|\left(q_{0}+k_{0}\right) S\left(w-w_{n}^{t}\right)\right] \\
= & M\left(q_{0}+k_{0}\right)\left[(1-t) S(w)+\left|t-t_{0}\right| S\left(w-w_{n}^{t}\right)\right],
\end{aligned}
$$

where $M=M_{3}\left(q_{0}, p_{0}, k_{0}, \alpha, K, D\right)$, and

$$
S(w) \leq M\left(k_{1}+k_{2}+k_{3}\right)=k,
$$

where $k_{1}, k_{2}, k_{3}$ are non-negative constants as stated in (1.3), (1.6) and (1.10). From (4.5) and (4.6), it follows

$$
\begin{aligned}
& S\left(w-w_{n+1}^{t}\right) \\
& \leq \gamma\left[(1-t) S(w)+\left|t-t_{0}\right| S\left(w-w_{n}^{t}\right)\right] \\
& \leq \gamma(1-t) S(w)\left(1+\gamma\left|t-t_{0}\right|+\gamma^{2}\left|t-t_{0}\right|^{2}+\cdots+\gamma^{n}\left|t-t_{0}\right|^{n}\right) \\
& +\gamma^{n+1}\left|t-t_{0}\right|^{n+1} S\left(w-w_{0}^{t}\right) \\
& \leq \gamma(1-t) S(w) \frac{1-\left(\gamma\left|t-t_{0}\right|\right)^{n+1}}{1-\gamma\left|t-t_{0}\right|} \\
& +\gamma^{n+1}\left|t-t_{0}\right|^{n+1} S\left(w-w_{0}^{t}\right),
\end{aligned}
$$

where $\gamma=M\left(q_{0}+k_{0}\right)$, and we choose that $w_{0}^{t}=w\left(z, t_{0}\right)$ is the solution of Problem $\mathrm{B}_{1}$ for (2.22) with $t=t_{0}$ and $A(z)=\left(1-t_{0}\right) F(z, 0,0)$. Due to $w-w_{0}^{t}$ is a solution of Problem $\mathrm{B}_{1}$ for the complex equation

$$
\begin{aligned}
& \left(w-w_{0}^{t}\right)_{\bar{z}} \\
= & F\left(z, w, w_{z}\right)-t_{0} F\left(z, w_{0}^{t}, w_{0 z}^{t}\right)-\left(1-t_{0}\right) F(z, 0,0) \\
= & t_{0}\left[F\left(z, w, w_{z}\right)-F\left(z, w_{0}^{t}, w_{0 z}^{t}\right)\right] \\
& +\left(1-t_{0}\right)\left[F\left(z, w, w_{z}\right)-F(z, 0,0)\right] \\
= & t_{0} Q\left(w-w_{0}^{t}\right)_{z}+A\left(w-w_{0}^{t}\right) \\
& +\left(1-t_{0}\right)\left[F\left(z, w, w_{z}\right)-F(z, 0,0)\right],
\end{aligned}
$$

hence

$$
\begin{aligned}
& S\left(w-w_{0}^{t}\right) \\
\leq & M\left(1-t_{0}\right)\left[q_{0} L_{p_{0}, 2}\left(w_{z}, \bar{D}\right)+k_{0} C(w, \bar{D})\right] \\
\leq & M\left(q_{0}+k_{0}\right)\left(1-t_{0}\right) S(w) \leq \gamma\left(1-t_{0}\right) k .
\end{aligned}
$$

Finally, we obtain

$$
\begin{aligned}
& S\left(w-w_{n+1}^{t}\right) \\
\leq & \gamma k\left[\frac{1-\gamma\left|t-t_{0}\right|^{n+1}}{1-\gamma\left|t-t_{0}\right|}(1-t)+\gamma k \gamma^{n+1}\left|t-t_{0}\right|^{n+1}\left(1-t_{0}\right)\right] \\
= & \gamma k\left[\frac{1-\gamma\left|t-t_{0}\right|^{n+1}}{1-\gamma\left|t-t_{0}\right|}(1-t)+\left(\gamma\left|t-t_{0}\right|\right)^{n+1}\left(1-t_{0}\right)\right],
\end{aligned}
$$

this shows that (4.1) holds. If the positive constant $\delta$ is small enough, so that when $\left|t-t_{0}\right| \leq \delta, \gamma\left|t-t_{0}\right|<1, \quad n$ is sufficiently large and $t$ is close to 1 , then the right hand side becomes small.

\section{REFERENCES}

[1] I. N. Vekua, "Generalized Analytic Functions," Pergamon, Oxford, 1962.

[2] G. C. Wen, "Linear and Nonlinear Elliptic Complex Equations," Shanghai Scientific and Technical Publishers, Shanghai, 1986.

[3] G. C. Wen and H. Begehr, "Boundary Value Problems for Elliptic Equations and Systems," Longman Scientific and Technical Company, Harlow, 1990.

[4] G. C. Wen, "Approximate Methods and Numerical Analysis for Elliptic Complex Equations," Gordon and Breach, Amsterdam, 1999.

[5] G. C. Wen, D. C. Chen and Z. L. Xu, "Nonlinear Complex Analysis and Its Applications," Mathematics Monograph Series 12, Science Press, Beijing, 2008.

[6] G. C. Wen, "Recent Progress in Theory and Applications of Modern Complex Analysis," Science Press, Beijing, 2010. 